KAIST-TH 99/03, hep-ph/9902292

\title{
String or $M$ Theory Axion as a Quintessence
}

\author{
Kiwoon Choi \\ Department of Physics, Korea Advanced Institute of Science and Technology \\ Taejon 305-701, Korea
}

\begin{abstract}
A slow-rolling scalar field ( $Q \equiv$ Quintessence) with potential energy $V_{Q} \sim$ $\left(3 \times 10^{-3} \mathrm{eV}\right)^{4}$ has been proposed as the origin of accelerating universe at present. We investigate the effective potential of $Q$ in the framework of supergravity model including the quantum corrections induced by generic (nonrenormalizable) couplings of $Q$ to the gauge and charged matter multiplets. It is argued that the Kähler potential, superpotential and gauge kinetic functions of the underlying supergravity model are required to be invariant under the variation of $Q$ with an extremely fine accuracy in order to provide a working quintessence potential. Applying these results for string or $M$-theory, we point out that the heterotic $M$-theory or Type I string axion can be a plausible candidate for quintessence if (i) it does not couple to the instanton number of gauge interactions not weaker than those of the standard model and (ii) the modulus partner $\operatorname{Re}(Z)$ of the periodic quintessence axion $\operatorname{Im}(Z) \equiv \operatorname{Im}(Z)+1$ has a large $\operatorname{VEV}: \operatorname{Re}(Z) \sim \frac{1}{2 \pi} \ln \left(m_{3 / 2}^{2} M_{\text {Planck }}^{2} / V_{Q}\right)$. It is stressed that such a large $\operatorname{Re}(Z)$ gives the gauge unification scale at around the phenomenologically favored value $3 \times 10^{16} \mathrm{GeV}$. To provide an accelerating universe, the quintessence axion should be at near the top of its effective potential at present, which requires a severe fine tuning of the initial condition of $Q$ and $\dot{Q}$ in the early universe. We discuss a late time inflation scenario based on the modular and $\mathrm{CP}$ invariance of the moduli effective potential, yielding the required initial condition in a natural manner if the Kähler metric of the quintessence axion superfield receives a sizable nonperturbative contribution.
\end{abstract}




\section{INTRODUCTION AND SUMMARY}

Recent measurements of the luminosity-red shift relation for Type Ia supernovae suggest that the Universe is accelerating and thus a large fraction of the energy density has negative pressure [1]. A nonvanishing cosmological constant (vaccum energy density) would be the simplest form of dark energy density providing the necessary negative pressure. However there is a widespread prejudice that the correct, if exists any, solution to the cosmological constant problem [2] will lead to an exactly vanishing vacuum energy density. For instance, within the framework of the Euclidean wormhole mechanism [3] which has been proposed sometime ago as a solution to the cosmological constant problem, the vacuum energy density appears to exactly vanish if all fields (except for the spacetime metric) were settled down at their true vacuum expectation values (VEVs).

Recently quintessence in the form of slow-rolling scalar field has been proposed as an alternative form of dark energy density with negative pressure [ [ 4 ]. The true minimum of the quintessence potential is presumed to vanish, i.e. $\left(V_{Q}\right)_{\min }=0$, however the present value of $Q$ is displaced from the true minimum, providing

$$
V_{Q} \sim\left(3 \times 10^{-3} \mathrm{eV}\right)^{4}
$$

and a negative pressure with the equation of state:

$$
\omega=\frac{p_{Q}}{\rho_{Q}}=\frac{\frac{1}{2} \dot{Q}^{2}-V_{Q}}{\frac{1}{2} \dot{Q}^{2}+V_{Q}} \lesssim-0.5,
$$

while obeying its equation of motion:

$$
\ddot{Q}+3 H \dot{Q}+\frac{\partial V_{Q}}{\partial Q}=0
$$

In order to have negative pressure, we then need the following slow-roll condition:

$$
\left|\frac{\partial V_{Q}}{\partial Q}\right| \lesssim\left|\frac{V_{Q}}{M_{P}}\right|,
$$

where $M_{P}=M_{\text {Planck }} / \sqrt{8 \pi}=2.4 \times 10^{18} \mathrm{GeV}$ denotes the reduced Planck scale. This slowroll condition indicates that the typical range of $Q$ is of order $M_{P}$ or at least not far below $M_{P}$.

Given the assumption of $\left(V_{Q}\right)_{\min }=0$, one can in principle compute the quintessence potential $V_{Q}$ once the particle physics model for $Q$ is given. However the required size of $V_{Q}$ is extremely small compared to any of the mass scales of particle physics, and thus an utmost question is how such a small $V_{Q}$ can arise from realistic particle physics models. It must be stressed that $V_{Q}$ is required to be small over a range of $Q$ which is essentially of order $M_{P}$, not only for a particular value of $Q$. As a result, unless there were a symmetry which would enforce $V_{Q}$ to be flat enough, $V_{Q}$ suffers from the fine tuning problem much more severe than the conventional cosmological constant problem associated with the smallness of the potential energy at its minimum [5]. In fact, the most natural candidate for a light scalar field whose typical range of variation is of order $M_{P}$ is the string or $M$-theory moduli 
multiplets describing the (approximately) degenerate string or $M$-theory vacua. It is thus quite tempting to look at the possibility that $Q$ corresponds to a certain combination of the string or $M$-theory moduli superfields. In this paper, we wish to explore this possibility and point out that the heterotic $M$-theory or Type I string axion can be a plausible candidate for quintessence if its modulus component has a large VEV and its Kähler metric receives a sizable nonperturbative contribution.

To make the discussion more rigorous, we provide in section II a careful analysis of the low energy effective potential of generic quintessence円 in the framework of 4-dimensional effective supergravity model which is presumed to describe the dynamics of $Q$ at high energy scales. We include both the perturbative and nonperturbative corections to $V_{Q}$ induced by generic (nonrenormalizable) couplings of $Q$ with the gauge and charged matter multiplets in the model. It is then argued that the Kähler potential $(K)$, superpotential $(W)$ and gauge kinetic functions $\left(f_{a}\right)$ of the underlying supergravity model are required to be invariant under the variation of $Q$ with an extremely fine accuracy, which implies as one of its consequences that non-derivative couplings of $Q$ are extremely suppressed and so there is no $Q$-mediated long range force. Also estimated are the size of $Q$-invariance breaking for various terms in $K, W$ and $f_{a}$ which would produce the correct value of $V_{Q}$.

Applying these results for string or $M$-theory, we show in section III that the heterotic $M$ theory or Type I string axion can be a plausible candidate for quintessence. The quintessence axion may correspond to the heterotic $M$-theory axion arising from the three form field of 11-dimensional supergravity on a manifold with boundary [6] or to the Type I string axion arising from the R-R two form field [7]. To avoid a too large potential energy, it is required that the quintessence axion does not couple to the instanton number of gauge interactions not weaker than those of the standard model, particularly not to those of the QCD and even stronger hidden sector gauge interactions. Then the effective potential of heterotic $M$-theory axion is mainly induced by the membrane instantons wrapping the 2-cycle of the internal 6-manifold and stretched along the 11-th dimension, while that of Type I string axion is induced by $D 1$ or $D 5$ instantons wrapping the 2 or 6 -cycle. The resulting axion potential is estimated as

$$
V_{Q} \sim e^{-2 \pi\langle\operatorname{Re}(Z)\rangle} m_{3 / 2}^{2} M_{P}^{2} \cos [2 \pi \operatorname{Im}(Z)],
$$

where $\operatorname{Re}(Z)$ is the modulus partner of the periodic quintessence axion $\operatorname{Im}(Z) \equiv \operatorname{Im}(Z)+1$ and $m_{3 / 2}$ is the gravitino mass. Thus the quintessence potential energy (1) is obtained if (i) $\operatorname{Re}(Z)$ takes a VEV significantly larger than the self dual value of order unity:

$$
\langle\operatorname{Re}(Z)\rangle \sim \frac{1}{2 \pi} \ln \left(m_{3 / 2}^{2} M_{P}^{2} / V_{Q}\right) \sim 32,
$$

and (ii) (one-loop) threshold corrections to the gauge coupling constants not weaker than those of the standard model are $Z$-independent, which would assure that the quintessence axion does not couple to the instanton numbers generating a too large $V_{Q}$.

\footnotetext{
${ }^{1}$ Some aspects of the quintessence potential was discussed recently in [5].

${ }^{2}$ Of course this is true only when the tree level gauge coupling constants are also $Z$-independent.
} 
As will be discussed in section IV, when combined with $\alpha_{G U T}=1 / 25$ and $M_{P}=2.4 \times 10^{18}$ $\mathrm{GeV}$, the quintessence axion potential (5) which is presumed to take the value of (1) fixes all the couplings and scales of the underlying model. Particularly it gives the gauge unification scale

$$
M_{G U T} \sim 1.3 \gamma \times 10^{16}\left(\frac{32}{\langle\operatorname{Re}(Z)\rangle}\right)^{1 / 2} \mathrm{GeV},
$$

where $\gamma$ is a model-dependent constant of order unity which will be defined later. It is then interesting to note that $\langle\operatorname{Re}(Z)\rangle \sim 32$ required for a quintessence axion determines $M_{G U T}$ at a value close to the phenomenologically favored $3 \times 10^{16} \mathrm{GeV}$.

One potential problem of the string or $M$-theoretic quintessence axion is that it does not satisfy the second slow-roll condition:

$$
\left|\frac{\partial^{2} V_{Q}}{\partial Q^{2}}\right| \lesssim\left|\frac{V_{Q}}{M_{P}^{2}}\right| .
$$

As is well known, for the case of inflation in the early universe, this additional slow-roll condition is essential for the accelerated expansion goes over many Hubble times. Unless this condition is satisfied, the quintessence axion can lead to an accelerating universe at present only when it has a very particular initial value in the early universe, i.e. the quintessence axion suffers from the fine-tuning problem of initial condition. As we will see, the canonical quintessence axion field is given by

$$
Q=M_{P} \sqrt{2\left\langle K_{0}^{\prime \prime}\right\rangle} \operatorname{Im}(Z),
$$

where

$$
K_{0}^{\prime \prime}=\frac{\partial^{2} K_{0}}{\partial Z \partial Z^{*}}
$$

denotes the Kähler metric of the quintessence axion superfield $Z$. The slow-roll condition (四) applied for the axion potential (5) then leads to

$$
|2 \pi \operatorname{Im}(Z)|_{\text {present }} \lesssim \frac{1}{2 \pi} \sqrt{\left\langle 2 K_{0}^{\prime \prime}\right\rangle} \ll 1
$$

since it is expected that $\left\langle K_{0}^{\prime \prime}\right\rangle \ll 1$ for the modulus VEV (6). This implies that at present the angular field $2 \pi \operatorname{Im}(Z)$ takes a value at near the top of its effective potential. Since $V_{Q}$ violates the second slow-roll condition (8), viz

$$
\left|\frac{\partial^{2} V_{Q}}{\partial Q^{2}}\right|=\left|\frac{2 \pi^{2}}{\left\langle K_{0}^{\prime \prime}\right\rangle} \frac{V_{Q}}{M_{P}^{2}}\right| \gg\left|\frac{V_{Q}}{M_{P}^{2}}\right|,
$$

and also $\operatorname{Im}(Z)=0$ corresponds to the maximum of $V_{Q}$, one needs a severe fine tuning of the initial values of $Q$ and $\dot{Q}$ in order to have the present value satisfying (10). It turns out that the degree of fine tuning is quite sensitive to $\left\langle K_{0}^{\prime \prime}\right\rangle$ :

$$
|2 \pi \operatorname{Im}(Z)|_{\text {initial }} \lesssim \exp \left(-0.5 m_{0} / H_{0}\right) \sim \exp \left(-3 / \sqrt{\left\langle K_{0}^{\prime \prime}\right\rangle}\right),
$$


where $H_{0} \approx V_{Q} / 3 M_{P}^{2}$ denotes the Hubble expansion rate at present and $m_{0}^{2}=\left|\partial^{2} V_{Q} / \partial Q^{2}\right|=$ $\left|2 \pi^{2} V_{Q} / M_{P}^{2}\left\langle K_{0}^{\prime \prime}\right\rangle\right|$. If the Kähler metric of $Z$ is dominated by the perturbative contribution,

we have $\sqrt{\left\langle K_{0}^{\prime \prime}\right\rangle} \approx 1 /\langle\operatorname{Re}(Z)\rangle \approx 3 \times 10^{-2}$. Such small value of the Kähler metric would lead to too severe fine tuning of the initial value of the quintessence axion, particularly when the quantum fluctuation of the axion field in the early universe is taken into account. However there can be a large nonperturbative contribution to the Kähler potential [8], which may be necessary to stabilize $\operatorname{Re}(Z)$ at the value $\sim 32$ which is significantly bigger than the self dual value $\sim 1$. If the stabilization of $\operatorname{Re}(Z)$ is achieved by the nonperturbative effects encoded in the Kähler potential, the Kähler metric $K_{0}^{\prime \prime}$ would be dominated by the nonperturbative contribution, so that $\sqrt{\left\langle K_{0}^{\prime \prime}\right\rangle}$ can be significantly bigger than the perturbative value $3 \times 10^{-2}$. In this case, the degree of the fine tuning of the initial conditions can be ameliorated enough to allow a cosmological scenario yielding the required initial condition (12). At the end of section IV, we discuss a late time inflation scenario based on the modular and CP invariance of the moduli effective potential, yielding the initial value of the quintessence axion satisfying (12) if the Kähler metric is enhanced by nonperturbative effects as

$$
\sqrt{\left\langle K_{0}^{\prime \prime}\right\rangle} \approx 9 \times 10^{-2}
$$

This scenario utilizes the specific property of string/M-theoretic quintessence axion $\operatorname{Im}(Z)$ that its potential is of the order of $\bar{V} e^{-2 \pi \operatorname{Re}(Z)}$ where $\bar{V}=\mathcal{O}\left(H^{2} M_{P}^{2}\right)$ over the period during which the expansion rate $H \gtrsim m_{3 / 2}$ and $\bar{V}=\mathcal{O}\left(m_{3 / 2}^{2} M_{P}^{2}\right)$ for the subsequent period with $H \ll m_{3 / 2}$. This allows that $\operatorname{Im}(Z)$ has a mass of the order of $H$ during the late inflation with $H \sim m_{3 / 2}$ and $\operatorname{Re}(Z) \sim 1$, while it becomes extremely light after the inflation since $\operatorname{Re}(Z)$ is rolled down to a large value at the end of inflation. So during the late inflation, $\operatorname{Im}(Z)$ rolls toward the minimum of the potential. If the axion potential is almost CP invariant, which would be the case when the scale of $\mathrm{CP}$ violation is low enough compared to $M_{P}$, the minimum of the axion potential during the late inflation would coincide with either the minimum or the maximum of the exponentially suppressed axion potential at the present. If it coincides to the maximum and also the Kähler metric is enhanced as (13), the latetime inflation can provide the desired initial condition (12) without any conflict with the known observational results.

\section{EFFECTIVE POTENTIAL OF GENERIC QUINTESSENCE}

Our starting point is the 4-dimensional $N=1$ effective supergravity model [9] which is presumed to describe the dynamics of $Q$ at high energy scales not far below $M_{P}$. The Kähler potential, superpotential and gauge kinetic functions of the model can be written as

$$
\begin{aligned}
K= & K_{0}\left(Z_{i}, Z_{i}^{*}\right)+Z_{\alpha \bar{\beta}}\left(Z_{i}, Z_{i}^{*}\right) C^{\alpha} C^{* \beta}+\left[\frac{1}{2} H_{\alpha \beta}\left(Z_{i}, Z_{i}^{*}\right) C^{\alpha} C^{\beta}\right. \\
& \left.+\frac{1}{2} X_{\alpha \beta \bar{\gamma}}\left(Z_{i}, Z_{i}^{*}\right) C^{\alpha} C^{\beta} C^{* \gamma}+\text { h.c. }\right]+\ldots, \\
W= & W_{0}\left(Z_{i}\right)+\frac{1}{3 !} Y_{\alpha \beta \gamma}\left(Z_{i}\right) C^{\alpha} C^{\beta} C^{\gamma}+\frac{1}{4 !} \Gamma_{\alpha \beta \gamma \delta}\left(Z_{i}\right) C^{\alpha} C^{\beta} C^{\gamma} C^{\delta}+\ldots \\
f_{a}= & f_{a 0}\left(Z_{i}\right)+\frac{1}{2} F_{a \alpha \beta}\left(Z_{i}\right) C^{\alpha} C^{\beta}+\ldots
\end{aligned}
$$


where $C^{\alpha}$ denote the gauge-charged light matter superfields whose VEVs are far below $M_{P}$ and $Z_{i}$ stand for generic gauge-singlet light multiplets including the fields with large VEVs of $\mathcal{O}\left(M_{P}\right)$. $Z_{i}$ may correspond to the string or $M$-theory moduli superfields and/or some gauge-invariant composite superfields made of strongly interacting hidden sector fields and/or others whose typical range of variation is of $\mathcal{O}\left(M_{P}\right)$. The quintessence $Q$ is included in the model as a certain combination of $Z_{i}$ and $Z_{i}^{*}$ :

$$
Q \in\left\{Z_{i}, Z_{i}^{*}\right\}
$$

The ellipses stand for the terms of higher order in $C^{\alpha}$, and the leading non-renormalizable terms (in power countings of $C^{\alpha}$ ) are explicitly written to illustrate their effects on $V_{Q}$. Note that unless specified we are using the standard supergravity unit with $M_{P}=1$ throughout this paper.

The superpotential $W_{0}\left(Z_{i}\right)$ is assumed to be induced by some (nonperturbative) supersymmetry (SUSY) breaking dynamics, e.g. hidden sector gaugino condensation, which is already integrated out. The auxiliary $F$-components of certain $Z_{i}$ develop non-zero VEVs:

$$
F_{i}=\left\langle e^{K_{0} / 2} D_{i} W_{0}\right\rangle=\mathcal{O}\left(m_{3 / 2} M_{P}\right)
$$

thereby breaking SUSY, where $D_{i} W_{0}=\partial_{i} W_{0}+W_{0} \partial_{i} K_{0}$ is the Kähler covariant derivative with $\partial_{i}=\partial / \partial Z_{i}$. Here we concentrate on the supergravity-mediated SUSY breaking scenario [9] yielding the soft SUSY breaking parameters (the soft scalar masses, gaugino masses, e.t.c.) of order the gravitino mass $m_{3 / 2}=\left\langle e^{K_{0} / 2}\left|W_{0}\right|\right\rangle$. For the case of gauge-mediation [10], the discussion will be modified but still leads to the same conclusion that $K, W$, and $f_{a}$ should be invariant under the variation of quintessence, i.e. $Q$-independent, with an extremely fine accuracy. Note that unless constrained by some symmetries the coefficients functions in $K, W$, and $f_{a}$ are generically $Q$-dependent, particularly when the terms of arbitrary order in $Q$ are taken into account. Those coefficient functions determine the (nonrenormalizable) couplings of $Q$ to the light gauge and charged matter multiplets. For instance, $Q^{n} F_{\mu \nu}^{a} F^{a \mu \nu} / M_{P}^{n}$ arises from a $Q$-dependent $\operatorname{Re}\left(f_{a}\right), Q^{n} F_{\mu \nu}^{a} \tilde{F}^{a \mu \nu} / M_{P}^{n}$ from a $Q$ dependent $\operatorname{Im}\left(f_{a}\right), Q^{n} C^{\alpha} \psi^{\beta} \psi^{\gamma} / M_{P}^{n}$ from a $Q$-dependent $Y_{\alpha \beta \gamma}$, and so on. At any rate, once the $Q$-invariance condition is fulfilled, non-derivative couplings of $Q$ are highly suppressed, and as a result $Q$ does not mediate any macroscopic force.

Obviously the tree level potential of $Q$ can be determined by $K_{0}$ and $W_{0}$ via the conventional supergravity potential:

$$
V_{0}=e^{K_{0}}\left[K^{i \bar{j}} D_{i} W_{0} D_{\bar{j}} W_{0}^{*}-3\left|W_{0}\right|^{2}\right] \sim m_{3 / 2}^{2} M_{P}^{2},
$$

where $K^{i \bar{j}}$ is the inverse of the Kähler metric $K_{i j}=\partial_{i} \partial_{\bar{j}} K_{0}$ and the typical range of $V_{0}$ under the variation of $Z_{i}=\mathcal{O}\left(M_{P}\right)$ is estimated to be of order $m_{3 / 2}^{2} M_{P}^{2}$. When quantum corrections are taken into account, generic (nonrenormalizable) couplings of $Q$ to charged gauge and matter multiplets contribute to $V_{Q}$. The leading quantum correction comes from the quadratically divergent one-loop potential [11] including the piece depending on $Z_{\alpha \bar{\beta}}$ and $\operatorname{Re}\left(f_{a}\right)$ :

$$
\delta V_{1}=\frac{\Lambda^{2}}{16 \pi^{2}} e^{K_{0}} D_{i} W_{0} D_{\bar{j}} W_{0}^{*} R^{i \bar{j}} \sim \frac{1}{16 \pi^{2}} m_{3 / 2}^{2} \Lambda^{2}
$$


where the cutoff scale $\Lambda$ is of order the messenger scale of SUSY breaking, which is essentially of $\mathcal{O}\left(M_{P}\right)$ for supergravity-mediated SUSY-breaking models,

$$
R^{i \bar{j}}=K^{i \bar{k}} K^{l \bar{j}} \partial_{l} \partial_{\bar{k}}\left[\ln \left(\operatorname{det} Z_{\alpha \bar{\beta}}\right)-\ln \left(\operatorname{det} \operatorname{Re}\left(f_{a b}\right)\right)\right],
$$

where $f_{a b}=f_{a} \delta_{a b}$, and the typical range of $\delta V_{1}$ is estimated to be of order $\frac{1}{16 \pi^{2}} m_{3 / 2}^{2} \Lambda^{2}$ for the variation of $Z_{i}=\mathcal{O}\left(M_{P}\right)$. This piece of one-loop potential arises from the well-known $V_{1}=\frac{1}{16 \pi^{2}} \Lambda^{2} \operatorname{Str}\left(M^{2}\right)$ where the mass matrix $M^{2}$ includes the soft SUSY breaking scalar and gaugino masses depending on $Z_{\alpha \bar{\beta}}$ and $\operatorname{Re}\left(f_{a}\right)$ [11].

$H_{\alpha \beta}$ and $X_{\alpha \beta \bar{\gamma}}$ in $K$ and the Yukawa couplings $Y_{\alpha \beta \gamma}$ in $W$ affect the potential energy at two-loop order. It has been noted that the two-loop supergraph of Fig. 1 leads to the quadratically divergent Yukawa-dependent potential energy [12]:

$$
\delta V_{2} \sim \frac{\Lambda^{2}}{\left(16 \pi^{2}\right)^{2}} e^{K_{0}}\left|D W_{0}\right|^{2}|Y|^{2} \sim \frac{|Y|^{2}}{\left(16 \pi^{2}\right)^{2}} m_{3 / 2}^{2} \Lambda^{2}
$$

where

$$
\begin{aligned}
& \left|D W_{0}\right|^{2}=K^{i \bar{j}} D_{i} W_{0} D_{\bar{j}} W_{0}^{*}, \\
& |Y|^{2}=Z^{\alpha \bar{\alpha}} Z^{\beta \bar{\beta}} Z^{\gamma \bar{\gamma}} Y_{\alpha \beta \gamma} Y_{\bar{\alpha} \bar{\beta} \bar{\gamma}}^{*} .
\end{aligned}
$$

for $Z^{\alpha \bar{\beta}}$ being the inverse of $Z_{\alpha \bar{\beta}}$. It has been noted also that the Kähler metric $\left(Z_{\alpha \bar{\beta}}\right)$ and soft SUSY-breaking scalar mass $\left(m_{\alpha \bar{\beta}}^{2}\right)$ of $C^{\alpha}$ receive quadratically divergent corrections depending upon $H_{\alpha \beta}$ and $X_{\alpha \beta \bar{\gamma}}[13]$ :

$$
\begin{aligned}
\delta Z_{\alpha \bar{\beta}} & =-\frac{\Lambda^{2}}{16 \pi^{2}} R_{\alpha \bar{\beta}} \\
\delta m_{\alpha \bar{\beta}}^{2} & =\frac{\Lambda^{2}}{16 \pi^{2}} e^{K_{0}}\left|W_{0}\right|^{2} R_{\alpha \bar{\beta}}
\end{aligned}
$$

where

$$
R_{\alpha \bar{\beta}}=Z^{\beta \bar{\alpha}} Z^{\gamma \bar{\gamma}} X_{\alpha \beta \bar{\gamma}} X_{\bar{\alpha} \bar{\beta} \gamma}^{*}-Z^{\gamma \bar{\gamma}} K^{i \bar{j}} \partial_{\bar{j}} H_{\alpha \gamma} \partial_{i} H_{\bar{\beta} \bar{\gamma}}^{*} .
$$

When combined together as the supergraph of Fig. 2, these one-loop corrections lead to the following quartically-divergent two-loop correction to the potential energy:

$$
\begin{aligned}
\delta V_{2}^{\prime} & \sim\left(\frac{\Lambda^{2}}{16 \pi^{2}}\right)^{2} e^{K_{0}}\left|W_{0}\right|^{2}\left(|X|^{2}+|\bar{\partial} H|^{2}\right) \\
& \sim \frac{|X|^{2}+|\bar{\partial} H|^{2}}{\left(16 \pi^{2}\right)^{2}} \frac{m_{3 / 2}^{2} \Lambda^{4}}{M_{P}^{2}}
\end{aligned}
$$

where

$$
\begin{aligned}
|X|^{2} & =Z^{\alpha \bar{\alpha}} Z^{\beta \bar{\beta}} Z^{\gamma \bar{\gamma}} X_{\alpha \beta \bar{\gamma}} X_{\bar{\alpha} \bar{\beta} \gamma}^{*} \\
|\bar{\partial} H|^{2} & =Z^{\alpha \bar{\alpha}} Z^{\beta \bar{\beta}} K^{i \bar{j}} \partial_{\bar{j}} H_{\alpha \beta} \partial_{i} H_{\bar{\alpha} \bar{\beta}}^{*} .
\end{aligned}
$$

Finally $\Gamma_{\alpha \beta \gamma \delta}$ and $F_{a \alpha \beta}$ contributes to the potential energy through the 3-loop supergraph of Fig. 3: 


$$
\begin{aligned}
\delta V_{3} & \sim \frac{1}{\left(16 \pi^{2}\right)^{3}} e^{K_{0}}\left(\Lambda^{4}\left|D W_{0}\right|^{2}|\Gamma|^{2}+\Lambda^{6}\left|\bar{D} W_{0}^{*} \partial F\right|^{2}\right) \\
& \sim \frac{1}{\left(16 \pi^{2}\right)^{3}}\left(\frac{m_{3 / 2}^{2} \Lambda^{4}}{M_{P}^{2}}|\Gamma|^{2}+\frac{m_{3 / 2}^{2} \Lambda^{6}}{M_{P}^{4}}|\partial F|^{2}\right),
\end{aligned}
$$

where $\left(\bar{D} W_{0}^{*} \partial F\right)_{\alpha \bar{\beta}}=K^{i \bar{j}} \partial_{i} F_{a \alpha \bar{\beta}} D_{\bar{j}} W_{0}^{*}$ and

$$
\begin{aligned}
& |\Gamma|^{2}=Z^{\alpha \bar{\alpha}} Z^{\beta \bar{\beta}} Z^{\gamma \bar{\gamma}} Z^{\delta \bar{\delta}} \Gamma_{\alpha \beta \gamma \delta} \Gamma_{\bar{\alpha} \bar{\beta} \bar{\gamma} \bar{\delta}}^{*}, \\
& |\partial F|^{2}=K^{i \bar{j}} Z^{\alpha \bar{\beta}} Z^{\beta \bar{\alpha}} \partial_{i} F_{a \alpha \beta} \partial_{\bar{j}} F_{a \bar{\alpha} \bar{\beta}}^{*} .
\end{aligned}
$$

So far, we have discussed the effective potential induced by the coefficient functions in $K$, $W$ and $f_{a}$ except for $\operatorname{Im}\left(f_{0 a}\right)$ whose VEV corresponds to the vacuum angle of the $a$-th gauge group. In the effective supergravity model under consideration, possible nonperturbative hidden sector gauge interactions were already integrated out, and their effects are encoded in the effective superpotential $W_{0}$ which is a function of the holomorphic hidden sector gauge kinetic functions. This means that the effective potential of the both real and imaginary parts of the gauge kinetic functions of strongly interacting hidden sector are already included in (16).

Let us now consider the effective potential of the vacuum angles of the standard model gauge group. Due to the asymtotic freedom, the potential energy of the QCD vacuum angle $8 \pi^{2} \operatorname{Im}\left(f_{Q C D}\right)$ is induced mainly by the low energy QCD dynamics at energy scales around $1 \mathrm{GeV}$ [14], and thus is unambiguously determined as

$$
V_{Q C D} \sim f_{\pi}^{2} m_{\pi}^{2} \cos \left[8 \pi^{2} \operatorname{Im}\left(f_{Q C D}\right)\right],
$$

where $m_{\pi}$ and $f_{\pi}$ denote the pion mass and decay constant, respectively. However the potential energy of the electroweak vacuum angle $8 \pi^{2} \operatorname{Im}\left(f_{E W}\right)$ is mainly due to the $S U(2)_{L}$ instantons at high energy scales around $M_{G U T}$. Since the multi-fermion vertex of the standard model fermions induced by the electroweak instanton violates both the baryon $(B)$ and lepton $(L)$ numbers, $\triangle B=\triangle L=3$, the resulting potential energy is suppressed by the insertions of small $B$ and $L$-violating couplings in addition to the suppressions by the semiclassical factor $e^{-2 \pi / \alpha_{G U T}}$ and the small SUSY breaking factor of order $m_{3 / 2}^{2}$. We thus have

$$
V_{E W} \sim \epsilon e^{-2 \pi / \alpha_{G U T}} m_{3 / 2}^{2} M_{P}^{2} \cos \left[\operatorname{Im}\left(8 \pi^{2} f_{E W}\right)\right],
$$

where the small factor $\epsilon$ includes $B$ and $L$ violating couplings obeying the selection rule $\triangle B=\triangle L=3$ together with small Yukawa-type couplings and loop factors which are necessary to close all fermion zero modes [15]. The size of $\epsilon$ is highly model-dependent, particularly on the $B, L$, and fermion chirality-changing couplings available at $M_{G U T}$.

Summing all the contributions discussed so far, the typical size of the quintessence potential $V_{Q}$ is estimated to be

$$
\begin{aligned}
V_{Q} \sim & m_{3 / 2}^{2} M_{P}^{2}\left[\frac{\delta \ln \left(K_{0}\right)}{\delta Q}+\frac{\delta \ln \left(W_{0}\right)}{\delta Q}+\frac{\Lambda^{2}}{16 \pi^{2} M_{P}^{2}}\left(\frac{\delta Z_{\alpha \bar{\beta}}}{\delta Q}+\frac{\delta \operatorname{Re}\left(f_{a}\right)}{\delta Q}\right)\right. \\
& +\frac{\Lambda^{2}}{\left(16 \pi^{2}\right)^{2} M_{P}^{2}} \frac{\delta|Y|^{2}}{\delta Q}+\frac{\Lambda^{4}}{\left(16 \pi^{2}\right)^{2} M_{P}^{4}}\left(\frac{\delta|X|^{2}}{\delta Q}+\frac{\delta|\bar{\partial} H|^{2}}{\delta Q}\right)+\frac{\Lambda^{4}}{\left(16 \pi^{2}\right)^{3} M_{P}^{4}} \frac{\delta|\Gamma|^{2}}{\delta Q}
\end{aligned}
$$




$$
\left.+\frac{\Lambda^{6}}{\left(16 \pi^{2}\right)^{3} M_{P}^{6}} \frac{\delta|\partial F|^{2}}{\delta Q}+\frac{f_{\pi}^{2} m_{\pi}^{2}}{m_{3 / 2}^{2} M_{P}^{2}} \frac{\delta \operatorname{Im}\left(f_{Q C D}\right)}{\delta Q}+\epsilon e^{-2 \pi / \alpha_{G U T}} \frac{\delta \operatorname{Im}\left(f_{E W}\right)}{\delta Q}\right] Q_{\mathrm{typ}},
$$

where $\delta G$ denotes the variation of the coefficient function $G$ for the quintessence variation $\delta Q$ and $Q_{\text {typ }}$ is the typical range of the quintessence variation. Note that (26) represents the variation of $V_{Q}$ under the variation of $Q$ specified by $Q_{\text {typ }}$. In order that $V_{Q} \sim\left(3 \times 10^{-3} \mathrm{eV}\right)^{4}$ over the range of $Q_{\text {typ }}$, its variation should not significantly exceed $\left(3 \times 10^{-3} \mathrm{eV}\right)^{4}$ over the same range. This requires that each term in (26) does not significantly exceed $\left(3 \times 10^{-3} \mathrm{eV}\right)^{4}$ over the range of $Q_{\text {typ }}$. Note that although there may be a significant cancellation between different terms for a particular value of $Q$, it is extremely difficult that the cancellation persists over the range of $Q_{\text {typ }}$ which is essentially of order $M_{P}$ [5]. Requiring each term in (26) not exceed $\left(3 \times 10^{-3} \mathrm{eV}\right)^{4}$, we find

$$
\begin{aligned}
& \frac{\delta \ln \left(K_{0}\right)}{\delta Q} \lesssim \frac{10^{-88}}{\kappa_{Q} \kappa_{3 / 2}}, \quad \frac{\delta \ln \left(W_{0}\right)}{\delta Q} \lesssim \frac{10^{-88}}{\kappa_{Q} \kappa_{3 / 2}} \\
& \frac{\delta Z_{\alpha \bar{\beta}}}{\delta Q} \lesssim \frac{10^{-81}}{\kappa_{Q} \kappa_{\Lambda} \kappa_{3 / 2}}, \quad \frac{\delta|\bar{\partial} H|^{2}}{\delta Q} \lesssim \frac{10^{-74}}{\kappa_{Q} \kappa_{\Lambda}^{2} \kappa_{3 / 2}} \\
& \frac{\delta|X|^{2}}{\delta Q} \lesssim \frac{10^{-74}}{\kappa_{Q} \kappa_{\Lambda}^{2} \kappa_{3 / 2}}, \quad \frac{\delta|Y|^{2}}{\delta Q} \lesssim \frac{10^{-79}}{\kappa_{Q} \kappa_{\Lambda} \kappa_{3 / 2}} \\
& \frac{\delta|\Gamma|^{2}}{\delta Q} \lesssim \frac{10^{-72}}{\kappa_{Q} \kappa_{\Lambda}^{2} \kappa_{3 / 2}}, \quad \frac{\delta|\partial F|^{2}}{\delta Q} \lesssim \frac{10^{-67}}{\kappa_{Q} \kappa_{\Lambda}^{3} \kappa_{3 / 2}} \\
& \frac{\delta \operatorname{Re}\left(f_{a}\right)}{\delta Q} \lesssim \frac{10^{-81}}{\kappa_{Q} \kappa_{\Lambda} \kappa_{3 / 2}}, \quad \frac{\delta \operatorname{Im}\left(f_{Q C D}\right)}{\delta Q} \lesssim \frac{10^{-42}}{\kappa_{Q} \kappa_{3 / 2}}
\end{aligned}
$$

where $\kappa_{Q}=Q_{\text {typ }} / M_{P}, \kappa_{\Lambda}=\left(\Lambda / 10^{16} \mathrm{GeV}\right)^{2}, \kappa_{3 / 2}=\left(m_{3 / 2}^{2} / \mathrm{TeV}\right)^{2}$, and all other quantities are defined in the supergravity unit with $M_{P}=1$, e.g. $Q, K_{0}, W_{0}$, and $X_{\alpha \beta \bar{\gamma}}$ correspond to $Q / M_{P}, K_{0} / M_{P}^{2}, W_{0} / M_{P}^{3}$, and $M_{P} X_{\alpha \beta \bar{\gamma}}$, respectively. Note that at least one of the variations is required to saturate its upper limit to produce the desired quintessence potential energy.

When expanded in powers of $C^{\alpha}$ and also written in the unit with $M_{P}=1$, all gaugeinvariant coefficient functions in $K, W$, and $f_{a}$ are expected to be of order unity except for $W_{0}$ which is of order $m_{3 / 2}$. Furthermore none of $\kappa_{Q}, \kappa_{\Lambda}$, and $\kappa_{3 / 2}$ can be small enough to significantly compensate the extremely small numerators in (27). Then the limits of (27) on the variations of coefficient functions imply that $K, W$ and $f_{a}$ must be invariant under the variation of $Q$, i.e. $Q$-independent, with an extremely fine accuracy. Note that terms of higher order in $C^{\alpha}$ which are not explicitly discussed here are similarly constrained to be $Q$-independent. At any rate, if this $Q$-invariance is satisfied, $Q$ behaves like a (pseudo) Goldstone boson having only derivative couplings and thus does not mediate any macroscopic force.

A possible breaking of the $Q$-invariance whose size was not estimated in (27) is the variation of the electroweak vacuum angle $8 \pi^{2} \operatorname{Im}\left(f_{E W}\right)$ which is constrained as

$$
\frac{\delta \operatorname{Im}\left(f_{E W}\right)}{\delta Q} \lesssim \frac{10^{-20}}{\epsilon \kappa_{Q} \kappa_{3 / 2}}
$$

Although quite sensitive to the physics at $M_{G U T}$, generically $\epsilon$ is suppressed by many powers of small Yukawa couplings, loop factors, and also small $B$ and/or $L$-violating couplings, and 
so it can be easily smaller than $10^{-20}$ 15]. This would suggest that $\operatorname{Im}\left(f_{E W}\right)$ can have a sizable $Q$-dependence, and so a sizable $Q$-coupling to the electromagnetic $F_{\mu \nu} \tilde{F}^{\mu \nu}$ which may lead to an interesting observable consequence [16]. However in view of the gauge coupling unification, i.e. $\left\langle\operatorname{Re}\left(f_{Q C D}\right)\right\rangle=\left\langle\operatorname{Re}\left(f_{E W}\right)\right\rangle$, it is highly unlikely that $\operatorname{Im}\left(f_{E W}\right)$ has a sizable $Q$-dependence, while $\operatorname{Im}\left(f_{Q C D}\right)$ is $Q$-independent as in (27). p

Let us close this section with a brief discussion of how plausible are some of the frequently used forms of $V_{Q}$ [4] in view of our discussions above.

(a) Exponential potential: $V_{Q} \sim M^{4} e^{-Q / f_{Q}}$. This form of $V_{Q}$ may arise from some nonperturbative dynamics when $Q$ corresponds to a dilaton [19]. At certain level, one may adjust the dynamics of the model to make this exponential potential to be of order $\left(3 \times 10^{-3} \mathrm{eV}\right)^{4}$. However usually the gauge coupling constants in the model are determined by the dilaton $\mathrm{VEV}$, which means $\delta \operatorname{Re}\left(f_{a}\right) / \delta Q$ is of order unity. Then the supergravity loop effects depending on $\delta \operatorname{Re}\left(f_{a}\right) / \delta Q$ (see Eq.(26)) yield a dilaton potential energy of order $\frac{1}{16 \pi^{2}} m_{3 / 2}^{2} \Lambda^{2}$ which is too large to be a quintessence potential energy.

(b) Inverse power law potential: $V_{Q} \sim M^{4+k} / Q^{k}$. This form of $V_{Q}$ may arise from a nonperturbative dynamics when $Q$ corresponds to a composite degree of freedom [19]. An attractive feature of this potential is that $M$ does not have to be too small compared to the particle physics mass scales, e.g. $M \gtrsim 10 \mathrm{GeV}$ for $k \geq 3$ and $Q \sim M_{P}$. However the inverse power law behavior is so easily upset either by the supersymmetry breaking dynamics yielding the soft mass term $\sim m_{3 / 2}^{2} Q^{2}$ or by the Planck scale dynamics which would generate a non-renormalizable term $\delta V_{Q} \sim m_{3 / 2}^{2} M_{P}^{2} Q^{n} / M_{P}^{n}$. If any of such terms is induced, which is extremely difficult to avoid, the variation of $V_{Q}$ under $\delta Q \sim M_{P}$ would be too large to provide a quintessence potential.

(3) Pseudo-Goldston boson potential: $V_{Q} \sim M^{4} \cos \left(Q / f_{Q}\right)$. This form of $V_{Q}$ arises when $Q$ corresponds to a (pseudo) Goldstone boson, which is perhaps the most plausible possibility in view of the $Q$-invariance conditions of (27) [17]. Obviously then the $Q$-invariance corresponds to the nonlinearly realized global symmetry associated with the Goldstone boson $Q$. Still the remained question is how the explicit breaking of the non-linear global symmetry can be so tiny as in (27), which is very nontivial to achieve in view of that global symmetries are generically broken by Planck scale physics [20]. In the next section, we argue that heterotic $M$-theory or Type I string axion can be a plausible candidate for the quintessence Goldstone boson with the tiny $Q$-invariance breaking induced by the membrane or $D$-brane instantons.

\footnotetext{
${ }^{3}$ A possible loophole [17 for this argument is the possibility of massless up quark which has been argued to be phenomenologically viable 18. In this case, the effective potential of the QCD vacuum angle $\operatorname{Im}\left(f_{Q C D}\right)$ is suppressed by $z=m_{u} / m_{d}$, i.e. $V_{Q C D} \sim z f_{\pi}^{2} m_{\pi}^{2}$, and then the upper bound on $\delta \operatorname{Im}\left(f_{Q C D}\right) / \delta Q$ in Eq.(27) should be read as $10^{-42} / z \kappa_{Q} \kappa_{\Lambda}$. Thus for $|z| \lesssim 10^{-40}, \operatorname{Im}\left(f_{Q C D}\right)$ can have a sizable $Q$-dependence and so $Q$ can couple to both $(F \tilde{F})_{Q C D}$ and $(F \tilde{F})_{E W}$.
} 


\section{HETEROTIC $M$ OR TYPE I STRING AXION AS QUINTESSENCE}

The most natural candidate for a light scalar field whose typical range of variation is of order $M_{P}$ is the string or $M$-theory moduli multiplets describing the (approximately) degenerate string or $M$-theory vacua. It is thus quite tempting to look at the possibility that $Q$ corresponds to a certain combination of the string or $M$-theory moduli superfields.

In this section, we examine this possibility and point out that the heterotic $M$-theory or Type I string axion can be a plausible candidate for quintessence. The moduli superfields of our interests are $Z_{I}=\left(S, T_{i}\right)$ including the axion components $\operatorname{Im}\left(Z_{I}\right)$ together with the modulus components $\operatorname{Re}\left(Z_{I}\right)$ which correspond to the string dilaton or the length of the 11th segment and the Kähler moduli describing the size and shape of the internal 6-manifold. Explicit computations show that generically

$$
\frac{\delta K_{0}}{\delta \operatorname{Re}\left(Z_{I}\right)}=\mathcal{O}(1),
$$

and so the effective potential of the modulus components are of order $m_{3 / 2}^{2} M_{P}^{2}$ and the modulus masses are of order $m_{3 / 2}$ in view of the analysis in the previous section. It thus appears that the modulus components $\operatorname{Re}\left(Z_{I}\right)$ can not provide a quintessence. However as we will see still quintessence can arise as a linear combination of the axion components $\operatorname{Im}\left(Z_{I}\right)$ if the corresponding modulus component has a large $\mathrm{VEV} \sim 32$.

Let us first consider the heterotic $M$-theory on a 11-dimensional manifold with boundary which is invariant under the $Z_{2}$-parity [6]:

$$
C \rightarrow-C, \quad x^{11} \rightarrow-x^{11}
$$

where $C=C_{A B C} d x^{A} d x^{B} d x^{C}$ is the 3 -form field in the 11-dimensional supergravity. When compactified to 4 -dimensions, axions arise as the massless modes of $C_{\mu \nu 11}$ and $C_{m n 11}$ :

$$
\epsilon^{\mu \nu \rho \sigma} \partial_{[\nu} C_{\rho \sigma] 11}=\partial^{\mu} \eta_{S}, \quad C_{m n 11}=\sum_{i} \eta_{i}\left(x^{\mu}\right) \omega_{m n}^{i}
$$

where $\omega^{i}\left(i=1\right.$ to $\left.h_{1,1}\right)$ form the basis of the integer $(1,1)$ cohomology of the internal 6 manifold and $\mu, \nu$ are tangent to the noncompact 4-dimensional spacetime. In 4-dimensional effective supergravity, these axions appear as the pseudo-scalar components of chiral multiplets:

$$
\begin{aligned}
& S=(4 \pi)^{-2 / 3} \kappa^{-4 / 3} V+i \eta_{S}, \\
& T_{i}=(4 \pi)^{-1 / 3} \kappa^{-2 / 3} \int_{\mathcal{C}_{i}} \omega \wedge d x^{11}+i \eta_{i},
\end{aligned}
$$

where $\kappa^{2}$ denotes the 11-dimensional gravitational coupling, $V$ is the volume of the internal 6-manifold with the Kähler two form $\omega$, and the integral is over the 11-th segment and also over the 2-cycle $\mathcal{C}_{i}$ dual to $\omega^{i}$. Here the axion components are normalized by the discrete Peccei-Quinn (PQ) symmetries:

$$
\operatorname{Im}(S) \rightarrow \operatorname{Im}(S)+1, \quad \operatorname{Im}\left(T_{i}\right) \rightarrow \operatorname{Im}\left(T_{i}\right)+1,
$$


which are the parts of discrete modular symmetries involving also the dualities between large and small $\operatorname{Re}(S)$ or $\operatorname{Re}\left(T_{i}\right)$.

Holomorphy and the discrete PQ symmetries imply that in the large $\operatorname{Re}(S)$ and $\operatorname{Re}\left(T_{i}\right)$ limits the gauge kinetic functions can be written as

$$
4 \pi f_{a}=k_{a} S+\sum_{i} l_{a i} T_{i}+\mathcal{O}\left(e^{-2 \pi S}, e^{-2 \pi T_{i}}\right),
$$

where $k_{a}$ and $l_{a i}$ are model-dependent quantized real constants and the exponentially suppressed terms are possibly due to the membrane or 5-brane instantons. For a wide class of compactifed heterotic $M$-theory, we have [22 26]

$$
\begin{aligned}
& 4 \pi f_{E_{8}}=S+\sum_{i} l_{i} T_{i}+\mathcal{O}\left(e^{-2 \pi S}, e^{-2 \pi T_{i}}\right), \\
& 4 \pi f_{E_{8}^{\prime}}=S-\sum_{i} l_{i} T_{i}+\mathcal{O}\left(e^{-2 \pi S}, e^{-2 \pi T_{i}}\right),
\end{aligned}
$$

where $l_{i} T_{i}$ corresponds to the one-loop threshold correction in perturbative heterotic string terminoloy with the quantized coefficients $l_{i}$ determined by the instanton numbers on the hidden wall and also the orbifold twists.]

Let $Q$ denote a linear combination of $\operatorname{Im}\left(T_{i}\right)$ for which the combination $\sum_{i} l_{i} T_{i}$ is invariant under

$$
U(1)_{Q}: \quad Q \rightarrow Q+\text { constant. }
$$

Note that such $Q$ exists always as long as $h_{1,1}>1$. In this regard, models of particular interest are the recently discovered threshold-free models with $l_{i}=0$ [26] for which any of $\operatorname{Im}\left(T_{i}\right)$ can be identified as $Q$. At any rate, once such $Q$ exists, we have

$$
\frac{\delta f_{a}}{\delta Q}=\mathcal{O}\left(e^{-2 \pi T}\right)
$$

where the internal 6-manifold is assumed to be isotropic and thus $\operatorname{Re}\left(T_{i}\right) \approx \operatorname{Re}(T)$ for all $T_{i}$. Holomorphy and discrete PQ symmetries imply also

$$
\frac{\delta Y_{\alpha \beta \gamma}}{\delta Q}=\mathcal{O}\left(e^{-2 \pi T}\right)
$$

for the Yukawa couplings (and also higher order holomorphic couplings) in the superpotential of (14). If $W_{0}$ is induced by hidden sector dynamics described by $Q$-independent gauge, Yukawa, and higher order holomorphic couplings, we have

\footnotetext{
4 This feature of the gauge kinetic function was first noted in [21].

${ }^{5}$ For compactifications on smooth Calabi-Yau space, $l_{i}$ can be determined either in the perturbative heterotic string limit [27,22] or in the 11-dimensional supergravity limit [28,25] and they take half-integer values [24], while they can be generic rational numbers for orbifolds [26].
} 


$$
\frac{\delta W_{0}}{\delta Q}=\frac{\delta W_{0}}{\delta f_{a}} \frac{\delta f_{a}}{\delta Q}+\frac{\delta W_{0}}{\delta Y_{\alpha \beta \gamma}} \frac{\delta Y_{\alpha \beta \gamma}}{\delta Q}+\ldots=\mathcal{O}\left(e^{-2 \pi T} W_{0}\right) .
$$

About the Kähler potential, the discrete PQ symmetries (33) imply that it can be written as $K=\sum_{n} K_{n} \exp \left[i 2 \pi n \operatorname{Im}\left(T_{i}\right)\right]$ where $K_{n}$ is a function of $\operatorname{Re}\left(T_{i}\right)$ and other moduli (and also of charged matter superfields). Obviously then $U(1)_{Q}$ is broken by $K_{n}$ with $n \neq 0$. To estimate the size of $K_{n}$, it is convenient to consider the 4-dimensional $N=1$ vacuum as a deformation of $N=2$ vacuum keeping the discrete PQ symmetries unbroken and also $\operatorname{Re}\left(T_{i}\right)$ at large values [24]. Note that this deformation is not a shift within the $N=2$ moduli space, but involves the shifts of massive fields breaking $N=2$ supersymmetry down to $N=1$ supersymmetry. At $N=2$ vacuum, $T_{i}$ correspond to the coordinates of a special Kähler manifold whose Kähler potential is determined by the holomorphic prepotential [29, 30], and thus the discrete PQ symmetries imply that $K_{n} \propto e^{-2 \pi n T_{i}}$. As long as the discrete PQ symmetries are kept unbroken and also $\operatorname{Re}\left(T_{i}\right)$ kept large, this exponential suppression of the $U(1)_{Q}$-breaking is expected to be maintained when $N=2$ vacuum is deformed to $N=1$ vacua of phenomenological interests, implying that

$$
\frac{\delta K}{\delta Q}=\mathcal{O}\left(e^{-2 \pi T}\right)
$$

even at $N=1$ vacua.

When applied for the effective potential analysis of the previous section, Eqs. (37), (38), (39), and (40) assure that in the large $\operatorname{Re}(T)$ limit the quintessence axion potential is exponentially suppressed as

$$
V_{Q} \sim e^{-2 \pi\langle\operatorname{Re}(T)\rangle} m_{3 / 2}^{2} M_{P}^{2} \cos [2 \pi \operatorname{Im}(T)]
$$

even when all possible quantum corrections are taken into account. Thus what is necessary and sufficient for this axion potential to be of order $\left(3 \times 10^{-3} \mathrm{eV}\right)^{4}$ is

$$
\langle\operatorname{Re}(T)\rangle \sim \frac{1}{2 \pi} \ln \left(m_{3 / 2}^{2} M_{P}^{2} / V_{Q}\right) \sim 32,
$$

where $m_{3 / 2} \sim 1 \mathrm{TeV}$ are used for numerical estimate. As we will see in the next section, this value of $\operatorname{Re}(T)$ can be compatible with $\alpha_{G U T} \sim 1 / 25$ only in the heterotic $M$ theory limit, not in the perturbative heterotic string limit. Although the above quintessence

6 This conclusion is supported by the observation that the nonlinear PQ transformation $U(1)_{i}$ : $\delta T_{i}=i c_{i}\left(c_{i}=\right.$ real constant $)$ originates from the local transformation of the 11-dimensional three form field, $\delta C=\omega^{i} \wedge d x^{11} \partial_{11} \beta^{i}\left(x^{11}\right)$ with $c_{i}=\int \delta C=\int \omega^{i} \wedge d x^{11} \partial_{11} \beta^{i}$. As was noted in [22], this suggests that $U(1)_{i}$ is broken only by nonperturbative effects defined on the boundaries or by those involving the bulk degree of freedoms wrapping the 2-cycle $\mathcal{C}_{i}$ dual to $\omega^{i}$ and stretched between the boundaries. Since $U(1)_{Q}$ was chosen to avoid the breaking by gauge instantons on the boundaries, it appears to be broken only by the stretched membrane instantons wrapping $\mathcal{C}_{i}$ whose effects are suppressed by $e^{-2 \pi T_{i}}$.

7 About stabilizing $\operatorname{Re}(T)$ at this large value, it has been shown in 31 that both $\operatorname{Re}(S)$ and $\operatorname{Re}(T)$ can be stabilized at large VEVs of order $\frac{1}{\alpha_{G U T}}$ by the combined effects of the multi-gaugino condensations and the membrane instantons wrapping the 3-cycle of the internal 6-manifold. 
axion potential has been obtained by the macroscopic analysis based on supersymmetry and discrete PQ symmetries, one can easily identify its microscopic origin by noting that $2 \pi \operatorname{Re}\left(T_{i}\right)=(4 \pi)^{-1 / 3} \kappa^{-2 / 3} \int_{\mathcal{C}_{i}} \omega \wedge d x^{11}$ corresponds to the Euclidean action of the membrane instanton wrapping $\mathcal{C}_{i}$ and stretched along the 11-th segment [22]. When extrapolated to the perturbative heterotic string limit, such membrane instanton corresponds to the heterotic string worldsheet instanton wrapping the same 2-cycle [32]. Explicit computations then show that the Kähler potential and/or the gauge kinetic functions are indeed corrected by worldsheet instantons, yielding $\delta K_{0}=\mathcal{O}\left(e^{-2 \pi T}\right)$ and $\delta f_{a}=\mathcal{O}\left(e^{-2 \pi T}\right)$ 33. These corrections can be smoothly extrapolated back to the heterotic $M$-theory limit and identified as the corrections induced by stretched membrane instantons. When integrated out, the correction to the hidden sector gauge kinetic function leaves its trace in the effective superpotential as $\delta W_{0}=\mathcal{O}\left(e^{-2 \pi T} W_{0}\right)$. The axion potential (41) is then obtained from the supergravity potential (16) with $\delta K_{0}=\mathcal{O}\left(e^{-2 \pi T}\right)$ and/or $\delta W_{0}=\mathcal{O}\left(e^{-2 \pi T} W_{0}\right)$.

Let us now turn to Type I string axions. The Type I axions (again normalized by the discrete PQ symmetries of (33)) correspond to the massless modes of the R-R two form fields $B_{\mu \nu}$ and $B_{m n}, \epsilon^{\mu \nu \rho \sigma} \partial_{\nu} B_{\rho \sigma}=\partial^{\mu} \eta_{S}, B_{m n}=\sum_{i} \eta_{i}\left(x^{\mu}\right) \omega_{m n}^{i}$, and form 4-dimensional chiral multiplets together with the string dilaton $e^{D}$ and the internal space volume $V$ :

$$
\begin{aligned}
& S=(2 \pi)^{-6} \alpha^{\prime-3} e^{-D} V+i \eta_{S}, \\
& T_{i}=(2 \pi)^{-2} \alpha^{\prime-1} e^{-D} \int_{\mathcal{C}_{i}} \omega+i \eta_{i} .
\end{aligned}
$$

Here we include $D 9$ and $D 5$ branes in the vaccum configuration, and consider the 4dimensional gauge couplings $\alpha_{9}$ and $\alpha_{5 i}$ defined on $D 9$ branes wrapping the internal 6manifold and $D 5$ branes wrapping the 2 -cycles $\mathcal{C}_{i}$, respectively [34]. Again holomorphy and discrete PQ symmetries imply that the corresponding gauge kinetic functions can be written as (34). A simple leading order calculation gives $\alpha_{9}=1 / \operatorname{Re}(S)$ and $\alpha_{5 i}=1 / \operatorname{Re}\left(T_{i}\right)$, and so 34

$$
4 \pi f_{9}=S, \quad 4 \pi f_{5 i}=T_{i} .
$$

However this leading order result can receive perturbative and/or non-perturbative corrections. Generic perturbative corrections can be expanded in powers of the string coupling $e^{D}$, the string inverse tension $\alpha^{\prime}$, and also the inverse tension $e^{D} \alpha^{\prime k}$ of $D_{2 k-1}$ branes [7]. Generically they scale as

$$
e^{n D} \alpha^{\prime m} \propto[\operatorname{Re}(T)]^{\frac{n+m}{2}}[\operatorname{Re}(S)]^{-\frac{3 n+m}{2}} .
$$

Combined with the leading order result (44) and also the general form of gauge kinetic function (34) dictated by holomorphy and discrete PQ symmetries, this scaling behavior implies that $f_{9}$ can receive a $T_{i}$-dependent correction at order $\alpha^{\prime 2}$, while there is no perturbative correction to $f_{5 i}$, and so

$$
\begin{aligned}
4 \pi f_{9} & =S+l_{i} T_{i}+\mathcal{O}\left(e^{-2 \pi S}, e^{-2 \pi T_{i}}\right), \\
4 \pi f_{5 i} & =T_{i}+\mathcal{O}\left(e^{-2 \pi S}, e^{-2 \pi T_{i}}\right) .
\end{aligned}
$$

Similarly to the case of heterotic $M$-theory axion, the quintessence $Q$ can arise as a linear combination of $\operatorname{Im}(S)$ and $\operatorname{Im}\left(T_{i}\right)$, however its explicit form depends on how the 
gauge couplings not weaker than those of the standard model are embedded in the model. Note that for such not-so-weak gauge couplings both $\operatorname{Re}\left(f_{a}\right)$ and $\operatorname{Im}\left(f_{a}\right)$ are required to be $Q$-independent as in (27), while for the weaker gauge interactions with $\alpha_{a}\left(M_{G U T}\right) \lesssim 1 / 32$, $\operatorname{Im}\left(f_{a}\right)$ are allowed to have a sizable $Q$-dependence. Here are some possibilities. If all of the not-so-weak gauge couplings are embedded in $\alpha_{5 i}, \operatorname{Im}(S)$ can be a quintessence when $\langle\operatorname{Re}(S)\rangle \sim 32$ which is necessary for $V_{Q} \sim\left(3 \times 10^{-3} \mathrm{eV}\right)^{4}$, and also the associated gauge coupling $\alpha_{9}\left(M_{G U T}\right) \lesssim 1 / 32$ which may be necessary to avoid a too large $V_{Q}$ induced by the gauge instantons of $\alpha_{9}$. If not-so-weak couplings are embedded in $\alpha_{9}$, any of $\operatorname{Im}\left(T_{i}\right)$ can be a quintessence when $\left\langle\operatorname{Re}\left(T_{i}\right)\right\rangle \sim 32$. If the vacuum does not include any $D 5$ brane wrapping the particular 2-cycle, e.g. the $i$-th cycle, the corresponding axion $\operatorname{Im}\left(T_{i}\right)$ can be a quintessence independently of the embedding when $\left\langle\operatorname{Re}\left(T_{i}\right)\right\rangle \sim 32$.

Once the quintessence component satisfying the constraints on gauge kinetic functions is identified, the $Q$-dependence of the Kähler potential and superpotential is suppressed by $e^{-2 \pi Z}(Z=S$ or $T)$ as in the case of heterotic $M$-theory, and so the quintessence axion potential is again given by (41) where now $T$ can be either $S$ or $T_{i}$. Microscopic origin of this Type I axion potential can be easily identified also by noting that $2 \pi \operatorname{Re}(S)$ corresponds to the Euclidean action of $D 5$ brane instanton wrapping the internal 6-manifold and $2 \pi \operatorname{Re}\left(T_{i}\right)$ is of $D 1$ string instanton wrapping the 2-cycle $\mathcal{C}_{i}$.

\section{COUPLINGS AND SCALES WITH QUINTESSENCE AXION}

In the previous section, it was noted that the heterotic $M$ or Type I string axion can provide a quintessence dark energy when its modulus partner has a VEV $\sim 32$. In this section, we discuss the couplings and scales associated with this modulus VEV.

\section{A. Couplings and Scales in Heterotic M-theory:}

For simplicity, let us consider a model without $T$-dependent threshold corrections, i.e. the quantized coefficients $l_{i}=0$ in Eq. (35). We then have [28,22]

$$
\begin{aligned}
& \frac{1}{\alpha_{G U T}}=(4 \pi)^{-2 / 3} \kappa^{-4 / 3} V=\operatorname{Re}(S), \\
& \omega=\frac{(4 \pi)^{1 / 3} \kappa^{2 / 3}}{\pi \rho} \sum_{i} \operatorname{Re}\left(T_{i}\right) \omega^{i}, \\
& V=\frac{1}{3 !} \int \omega \wedge \omega \wedge \omega \approx \frac{4 \pi \kappa^{2}}{(\pi \rho)^{3}} \frac{\sum_{i j k} C_{i j k}}{6}[\operatorname{Re}(T)]^{3}
\end{aligned}
$$

where $\pi \rho$ is the length of the 11-th segment, $\omega$ is the Kähler form of the internal 6-manifold, and $C_{i j k}=\int \omega^{i} \wedge \omega^{j} \wedge \omega^{k}$ are the intersection numbers of the integer $(1,1)$ cohomoloy basis $\omega^{i}$. Here we have used the isotropy condition $\operatorname{Re}\left(T_{i}\right) \approx \operatorname{Re}(T)$. Combining these with

$$
M_{P}^{2}=\pi \rho V / \kappa^{2},
$$

one easily finds

$$
\begin{aligned}
& M_{K K} \equiv \frac{\gamma}{V^{1 / 6}}=\frac{\gamma M_{P}}{2 \sqrt{\pi}\left(\sum C_{i j k} / 6\right)^{1 / 6}[\operatorname{Re}(S)]^{1 / 2}[\operatorname{Re}(T)]^{1 / 2}} \\
& \kappa^{-2 / 3}(\pi \rho)^{3}=(4 \pi)^{1 / 3}\left(\frac{\sum C_{i j k}}{6}\right) \frac{[\operatorname{Re}(T)]^{3}}{\operatorname{Re}(S)}
\end{aligned}
$$


where $M_{K K}$ denotes the Kaluza-Klein threshold mass scale for the internal 6-manifold with volume $V$. Here $\gamma$ is a constant of order unity whose precise value depends on the details of compactification and also on the regularization of the threshold effects due to massive Kaluza-Klein modes. (For instance, $\gamma$ is expected to be around $2 \pi$ for toroidal compactifications, however it can be smaller for more complicate compactifications, e.g. about $\sqrt{2 \pi}$ for the compactification on $S^{6}$.)

In heterotic $M$-theory, $M_{K K}$ can be identified as the unification scale of gauge couplings on the boundary [28]:

$$
M_{G U T}=M_{K K}
$$

Also the VEVs of $\operatorname{Re}(S)$ and $\operatorname{Re}(T)$ can be determined by the two phenomenological inputs: $\alpha_{G U T}=1 / 25$ and $V_{Q}=\left(3 \times 10^{-3} \mathrm{eV}\right)^{4}$, yielding

$$
\begin{aligned}
& \operatorname{Re}(S)=\frac{1}{\alpha_{G U T}} \sim 25 \\
& \operatorname{Re}(T)=\frac{1}{2 \pi} \ln \left(m_{3 / 2}^{2} M_{P}^{2} / V_{Q}\right) \sim 32 .
\end{aligned}
$$

Applying these moduli VEVs to (48), we find

$$
M_{G U T}=\frac{1.3 \gamma}{\left(\sum C_{i j k} / 6\right)^{1 / 6}} \times 10^{16} \mathrm{GeV}
$$

which is very close to the phenomenologically favored value $3 \times 10^{16} \mathrm{GeV}$. We stress that the large $\langle\operatorname{Re}(T)\rangle \sim 32$ required for the quintessence axion is essential for $M_{G U T}$ to have a value close to the phenomenologically favored value. Large $\operatorname{Re}(T)$ leads also to the length of the 11-th segment significantly bigger than the 11-dimensional Planck length:

$$
\pi \rho \sim 10 \kappa^{2 / 9}
$$

implying that we are indeed in the 11-dimensional heterotic $M$-theory limit corresponding to the strong coupling limit of the heterotic $E_{8} \times E_{8}^{\prime}$ string theory.

B. Couplings and Scales in Type I string with D9 and D5-branes:

Again for simplicity consider a model without $T$-dependent threshold correction to $f_{9}$ in (46). We then have [7]

$$
\begin{aligned}
& M_{P}^{2}=2(2 \pi)^{-7} \alpha^{\prime-4} e^{-2 D} V, \\
& \frac{1}{\alpha_{9}}=(2 \pi)^{-6} \alpha^{\prime-3} e^{-D} V=\operatorname{Re}(S), \\
& \frac{1}{\alpha_{5 i}}=(2 \pi)^{-2} \alpha^{\prime-1} e^{-D} \int_{\mathcal{C}_{i}} \omega=\operatorname{Re}\left(T_{i}\right),
\end{aligned}
$$

and so

$$
\begin{aligned}
& e^{2 D}=\frac{\operatorname{Re}(S)}{\left(\sum_{i j k} C_{i j k} / 6\right)[\operatorname{Re}(T)]^{3}}, \\
& M_{K K} \equiv \frac{\gamma}{V^{1 / 6}}=\frac{\gamma M_{P}}{2 \sqrt{\pi}\left(\sum C_{i j k} / 6\right)^{1 / 6}[\operatorname{Re}(S)]^{1 / 2}[\operatorname{Re}(T)]^{1 / 2}} \\
& M_{\text {string }} \equiv \frac{1}{\sqrt{\alpha^{\prime}}}=\frac{\sqrt{\pi} M_{P}}{\left(\sum C_{i j k} / 6\right)^{1 / 2}[\operatorname{Re}(S)]^{1 / 4}[\operatorname{Re}(T)]^{3 / 4}} .
\end{aligned}
$$


In Type I case with a quintessence axion $\operatorname{Im}(T)$ or $\operatorname{Im}(S)$, we also have

$$
\begin{aligned}
& M_{G U T}=M_{K K} \text { or } M_{\text {string }}, \\
& \frac{1}{\alpha_{G U T}}=\operatorname{Re}(S) \text { or } \operatorname{Re}(T) \sim 25, \\
& \frac{1}{2 \pi} \ln \left(m_{3 / 2}^{2} M_{P}^{2} / V_{Q}\right)=\operatorname{Re}(T) \text { or } \operatorname{Re}(S) \sim 32,
\end{aligned}
$$

depending upon how the standard model gauge couplings are embedded [34]. For these moduli VEVs,

$$
e^{2 D} \ll 1, \quad \alpha^{\prime} M_{K K}^{2}=\mathcal{O}(1)
$$

indicating that we are in a domain of weakly coupled string but of strongly coupled worldsheet sigma model. It is also straightforward to see that they also give $M_{G U T}$ which is very close to the phenomenologically favored value, similarly to the case of heterotic $M$-theory.

C. Quintessence Axion Scale and the Fine Tuning Problem of Initial Condition:

Let $K_{0}\left(Z+Z^{*}\right)$ denote the (dimensionless) Kähler potential of the quintessence axion multiplet $Z=T$ or $S$. When all massive moduli (including $\operatorname{Re}(Z)$ ) are integrated out, the effective lagrangian of the superlight quintessence axion is given by

$$
\begin{aligned}
\mathcal{L}_{\text {eff }} & =M_{P}^{2}\left\langle K_{0}^{\prime \prime}\right\rangle\left[\partial_{\mu} \operatorname{Im}(Z)\right]^{2}+m_{3 / 2}^{2} M_{P}^{2} e^{-2 \pi\langle\operatorname{Re}(Z)\rangle} \cos [2 \pi \operatorname{Im}(Z)] \\
& =\frac{1}{2}\left(\partial_{\mu} Q\right)^{2}+m_{3 / 2}^{2} M_{P}^{2} e^{-2 \pi\langle\operatorname{Re}(T)\rangle} \cos \left(Q / f_{Q}\right)
\end{aligned}
$$

where the canonical quintessence axion $Q$ and its decay constant $f_{Q}$ are given by

$$
Q=M_{P} \sqrt{2\left\langle K_{0}^{\prime \prime}\right\rangle} \operatorname{Im}(Z), \quad f_{Q}=\frac{1}{2 \pi} M_{P} \sqrt{2\left\langle K_{0}^{\prime \prime}\right\rangle}
$$

with the Kähler metric $K_{0}^{\prime \prime}=\partial^{2} K_{0} / \partial Z \partial Z^{*}$.

In order to have negative pressure, $Q$ must roll slowly and so satisfy

$$
\left|\frac{\partial V_{Q}}{\partial Q}\right| \lesssim\left|\frac{V_{Q}}{M_{P}}\right| .
$$

When applied for (54), this slow-roll condition leads to

$$
\begin{aligned}
|2 \pi \operatorname{Im}(Z)|_{\text {present }} & \lesssim \sqrt{2\left\langle K_{0}^{\prime \prime}\right\rangle} / 2 \pi \\
|2 \pi \operatorname{Im}(\dot{Z})|_{\text {present }} & \lesssim H_{0} / \sqrt{2\left\langle K_{0}^{\prime \prime}\right\rangle},
\end{aligned}
$$

implying that at present the angular field $2 \pi \operatorname{Im}(Z)$ should be at near the top of its effective potential unless the Kähler metric $K_{0}^{\prime \prime}$ is significantly bigger than the unity. (Here $H_{0}$ denotes the present Hubble expansion rate.)

At leading order approximation in string or $M$ theory, we have [0]

$$
K_{0} \approx-c \ln \left(Z+Z^{*}\right)
$$


where $c$ is a constant of order unity. Obviously this gives a small Kähler metric $K_{0}^{\prime \prime} \sim$ $1 /[\operatorname{Re}(Z)]^{2} \ll 1$ for $\operatorname{Re}(Z) \sim 32$. This then leads to

$$
\left|\frac{\partial^{2} V_{Q}}{\partial Q^{2}}\right|=\left|\frac{2 \pi^{2}}{\left\langle K_{0}^{\prime \prime}\right\rangle} \frac{V_{Q}}{M_{P}^{2}}\right| \gg\left|\frac{V_{Q}}{M_{P}^{2}}\right|,
$$

and thus the quintessence axion in string or $M$ theory largely violates the second slow-roll condition:

$$
\left|\frac{\partial^{2} V_{Q}}{\partial Q^{2}}\right| \lesssim\left|\frac{V_{Q}}{M_{P}^{2}}\right|
$$

As a consequence, the quintessence axion can lead to an accelerating universe at present, i.e. can satisfy Eq. (57), only when it has a very particular initial value in the early universe, i.e. the quintessence axion suffers from the fine-tuning problem of the initial conditions of $Q$ and $\dot{Q}$ in the early universe. As long as $\sqrt{\left\langle K_{0}^{\prime \prime}\right\rangle} / \pi \ll 1$, the corresponding quintessence axion requires the fine tuning of initial condition, however the degree of required fine tuning is quite sensitive to the value of $\sqrt{\left\langle K_{0}^{\prime \prime}\right\rangle}$. Although $K_{0}^{\prime \prime} \ll 1$ at leading order perturbation theory, it can be significantly enhanced by $\operatorname{Im}(Z)$-independent nonperturbative effects which would be responsible for stabilizing $\operatorname{Re}(Z)$ at large value $\sim 32$ [8]. In this case, the fine tuning problem of initial conditions would be ameliorated, however it is hard to imagine that nonperturbative effects are strong enough to give $K_{0}^{\prime \prime} \sim \pi^{2}$, so that allow to avoid the fine tuning of initial condition.

The above discussed fine tuning problem of initial conditions appears to be a serious difficulty of the quintessence axion. Here we present a late time inflation scenario which would resolve this difficulty for a reasonably enlarged value of the Kähler metric $\left\langle K_{0}^{\prime \prime}\right\rangle$. The gauge symmetries of string or $M$-theory include discrete modular group [7] under which $Z$ and other generic moduli $\Phi$ transform as

$$
\operatorname{Re}(Z) \rightarrow \frac{1}{\operatorname{Re}(Z)}, \quad \operatorname{Im}(Z) \rightarrow \operatorname{Im}(Z)+1, \quad \Phi \rightarrow \Phi^{\prime},
$$

and also CP [35] under which

$$
Z \rightarrow Z^{*}, \quad \Phi \rightarrow \Phi^{*}
$$

Here we take the simplest form of the $Z$-duality $(Z=S$ or $T)$, i.e. $\operatorname{Re}(Z) \rightarrow 1 / \operatorname{Re}(Z)$ with the self-dual value $\operatorname{Re}(Z)=1$, however our discussion is valid for other forms of the $Z$-duality transformation as long as the self-dual value is of order unity.

The modular and CP invariances assure that the invariant points,

$$
\operatorname{Re}(Z)=1, \quad \operatorname{Im}(Z)=0 \text { or } \frac{1}{2}, \quad \Phi=\Phi^{*} \text { or } \Phi^{\prime *},
$$

correspond to the stationary points of the effective action [36]. It is then quite possible that $Z=1$ was a local minimum of the effective potential during the inflation period if the inflaton is moduli invariant field. At this inflationary minimum, all the moduli masses have the same order of magnitude: 


$$
m_{\operatorname{Re}(Z)} \sim m_{\operatorname{Im}(Z)} \sim m_{\Phi} \sim H_{\mathrm{inf}}
$$

where $H_{\mathrm{inf}}=\sqrt{V_{\mathrm{inf}} / M_{P}^{2}}$ is the expansion rate during inflation. Note that the axion mass of $m_{\operatorname{Im}(Z)}$ is comparable to other moduli masses for $\langle\operatorname{Re}(Z)\rangle=\mathcal{O}(1)$. The modulus $\operatorname{Re}(Z)$ can have appropriate couplings to inflaton field, so that if inflaton takes its present value, $\operatorname{Re}(Z)=1$ becomes an unstable point of the moduli potential. Then after the inflation $\operatorname{Re}(Z)$ moves toward the present minimum at $\operatorname{Re}(Z) \sim 32$ for which

$$
m_{\operatorname{Re}(Z)} \sim m_{3 / 2}, \quad m_{\operatorname{Im}(Z)} \sim e^{-\pi\langle\operatorname{Re}(Z)\rangle} m_{3 / 2} .
$$

About the location of the minimum in the axion direction, we have just two possibilities if $\mathrm{CP}$ is not spontaneously broken in the moduli sector. One of the two CP invariant points, $\operatorname{Im}(Z)=0$ and $1 / 2$, is the minimum, while the other corresponds to the maximum. Our key assumption on the axion potential is that $\operatorname{Im}(Z)=0$ is the minimum for the inflationary modulus value $\operatorname{Re}(Z) \sim 1$, however it becomes the maximum for the present value $\operatorname{Re}(Z) \sim 32$. Note that the coefficient of the cosine potential of $\operatorname{Im}(Z)$ is a function of $\operatorname{Re}(Z)$, and so its sign can be changed when $\operatorname{Re}(Z)$ varies from the inflationary value to the present value.

Given the properties of the moduli potential discussed above, the cosmological scenario yielding the present values of $\operatorname{Im}(Z)$ and $\operatorname{Im}(\dot{Z})$ satisfying Eq. (57) goes as follows. There was a relatively late inflationary period in the early universe during which $\operatorname{Re}(Z)$ and other moduli were settled down near at the modular and CP-invariant local minimum of the potential, i.e. at $\langle\operatorname{Re}(Z)\rangle_{\mathrm{inf}}=1,\langle\operatorname{Im}(Z)\rangle_{\mathrm{inf}}=0$, and $\langle\Phi\rangle_{\mathrm{inf}}=\left\langle\Phi^{*}\right\rangle_{\text {inf }}$. Since the modulus $\operatorname{Re}(Z)$ is significantly away from the present value $\sim 32$, it is expected that the Hubble expansion rate $H_{\text {inf }}$ of this late inflation is at least of order $m_{3 / 2}$. To avoid a too large quantum fluctuation of the quintessence axion, we assume the minimal value

$$
H_{\mathrm{inf}}=\mathcal{O}\left(m_{3 / 2}\right) .
$$

During the inflation period, all moduli masses including that of $\operatorname{Im}(Z)$ are of order $H_{\text {inf }}$, and thus all moduli are settled down at the values sufficiently near at the minimum if the number of inflation efoldings $\left(N_{e}\right)$ are large enough [37]. In particular, just after the inflation, we have

$$
\begin{aligned}
& |2 \pi \operatorname{Im}(Z)|_{\text {inflation }}=\mathcal{O}\left(e^{-3 N_{e} / 2}\right)+\mathcal{O}\left(H_{\text {inf }} / M_{P}\right), \\
& |2 \pi \operatorname{Im}(\dot{Z})|_{\text {inflation }}=\mathcal{O}\left(e^{-3 N_{e} / 2} H_{\text {inf }}\right)+\mathcal{O}\left(H_{\text {inf }}^{2} / M_{P}\right),
\end{aligned}
$$

where the exponential suppression in the right hand side is due to the classical evolution toward the minimum of $V_{\text {inflation, }}$, while the second terms denote the effects of quantum fluctuations. After the inflation, $\operatorname{Re}(Z)$ moves toward the present value $\operatorname{Re}(Z) \sim 32$. After $\operatorname{Re}(Z)$ is settled down at its present value, $\operatorname{Im}(Z)=0$ becomes the maximum of the axion potential with

$$
m_{0} \equiv\left|\frac{\partial^{2} V_{Q}}{\partial Q^{2}}\right|_{Q=0}^{1 / 2} \approx \frac{\sqrt{3} \pi}{\sqrt{\left\langle K_{0}^{\prime \prime}\right\rangle}} H_{0}
$$

Since $m_{0} \gg H_{0}$, the inflationary value $\operatorname{Im}(Z) \sim 0$ is highly unstable againt the cosmological evolution during the period with $H_{0} \lesssim H \lesssim m_{0}$. The resulting unstability factor is given 
by $e^{\alpha m_{0} / H_{0}}$ where $\alpha$ is a constant of order unity whose precise value depends upon the initial conditions. More detailed study [38 suggests $\alpha \approx 0.5$ for the most of interesting initial conditions. In order for the quintessence axion to provide an accelerating universe at present, we then need

$$
\begin{aligned}
|2 \pi \operatorname{Im}(Z)|_{\text {inflation }} & \lesssim e^{-0.5 m_{0} / H_{0}} \sim e^{-3 / \sqrt{\left\langle K_{0}^{\prime \prime}\right\rangle}} \\
|2 \pi \operatorname{Im}(\dot{Z})|_{\text {inflation }} & \lesssim H_{\text {inf }} e^{-0.5 m_{0} / H_{0}} \sim H_{\text {inf }} e^{-3 / \sqrt{\left\langle K_{0}^{\prime \prime}\right\rangle}}
\end{aligned}
$$

In order for the initial condition (67) set by the late time inflation to satisfy the above condition, we need first a large efolding:

$$
N_{e} \gtrsim \frac{2}{\sqrt{\left\langle K_{0}^{\prime \prime}\right\rangle}},
$$

and also a small quantum fluctuation of the quintessence axion field during (and also after) the late inflation:

$$
\delta Q \sim \frac{H_{\mathrm{inf}}}{2 \pi} \sim \frac{m_{3 / 2}}{2 \pi} \lesssim M_{P} e^{-3 / \sqrt{\left\langle K_{0}^{\prime \prime}\right\rangle}}
$$

In fact, $N_{e}$ can not be arbitrarily large. For late time inflation with $H_{\text {inf }} \sim m_{3 / 2}$, it is very difficult to generate the observed density fluctuation $\delta \rho / \rho \sim 10^{-5}$. It is thus reasonable to assume that density fluctuations were created before the late inflation. Then the late inflation is required not to destroy the pre-exisiting density fluctuations. This consideration gives an upper bound of $N_{e}$ [37], yielding

$$
N_{e} \lesssim 25 \sim 30
$$

for a weak scale $m_{3 / 2}$ and the reheat temperature range $T_{r}=10^{5} \sim 10^{-2} \mathrm{GeV}$.

The above conditions (70), (71) and (72) can not be simultaneously satisfied for the perturbative value of $\left\langle K_{0}^{\prime \prime}\right\rangle$. At leading order in perturbation theory, we have $K_{0} \sim 3 \ln (Z+$ $Z^{*}$ ) and thus $\sqrt{\left\langle K_{0}^{\prime \prime}\right\rangle} \sim 1 /\langle\operatorname{Re}(Z)\rangle \sim 3 \times 10^{-2}$ for $\langle\operatorname{Re}(Z)\rangle \sim 32$, which is obvioulsy in conflict with the above conditions. However as was noted in the [8], there can be a rather large nonperturbative contribution to the Kähler potential which may be essential for stabilizing $\operatorname{Re}(Z)$ at the desired value $\sim 32$. In particular, if the nonperturbative terms involve a large power of $\left(Z+Z^{*}\right)$, e.g. $K_{n p}=h\left(Z+Z^{*}\right)^{k} e^{-b \sqrt{Z+Z^{*}}}=\mathcal{O}(1)$ with $k \gtrsim 6$, the Kähler metric can be significantly enlarged to a value

$$
\sqrt{\left\langle K_{0}^{\prime \prime}\right\rangle} \approx 9 \times 10^{-2}
$$

which would accomodate all the conditions for the quintessence axion to lead to an accelerating universe at present.

Acknowledgments: I thank K. Hwang for drawing the figures and H. B. Kim and S. Thomas for useful discussions. This work is supported in part by KOSEF Grant 981-0201004-2, KOSEF through CTP of Seoul National University, KRF under the Grant 1998-015D00071 and the Distinguished Scholar Exchange Program. 


\section{REFERENCES}

[1] S. Perlmutter et al., Nature 391, 51 (1998); A. G. Riess et al., astro-ph/9805201.

[2] For a review, see S. Weinberg, Rev. Mod. Phys. 61, 1 (1989).

[3] S. Coleman, Nucl. Phys. B310, 643 (1988).

[4] M. Özer and M. O. Taha, Nucl. Phys. B287, 797 (1987); B. Ratra and P. J. E. Peebles, Phys. Rev. D37, 3406 (1988); J. A. Frieman, C. T. Hill and R. Watkins, Phys. Rev. D46, 1226 (1992); M. S. Turner and M. White, Phys. Rev. D56, 4439 (1997); T. Chiba, N. Sugiyama and T. Nakamura, Mon. Not. R. Astron. Soc. 289, 5 (1997); ibid. 301, 72 (1998); R. R. Caldwell, R. Dave and P. J. Steinhardt, Phys. Rev. Lett. 80, 1582 (1998); L. Wang and P. J. Steinhardt, astro-ph/9804015; G. Huey et al., astro-ph/9804285; I. Zlater, L. Wang and P. J. Steinhardt, astro-ph/9807002; D. Huterer and M. S. Turner, astro-ph/9808133, T. Chiba and T. Nakamura, Prog. Theor. Phys. 100, 1077 (1998); T. Nakamura and T. Chiba, astro-ph/9810447.

[5] C. Kolda and D. H. Lyth, hep-ph/9811375.

[6] P. Horava and E. Witten, Nucl. Phys. B460, 506 (1996); Phys. Rev. D54, 7561 (1996).

[7] J. Polchinski, String Theory (Cambridge University Press, 1998).

[8] T. Banks and M. Dine, Phys. Rev. D50, 7454 (1994); J. A. Casas, Phys. Lett. B384, 103 (1996); K. Choi, H. B. Kim and H. D. Kim, Mod. Phys. Lett. A14, 125 (1999).

[9] For a review, see H. P. Nilles, Phys. Rep. 110, 1 (1984).

[10] For a review, see G. F. Giudice and R. Rattazzi, hep-ph/9801271.

[11] M. T. Grisaru, M. Rocek and A. Karlhede, Phys. Lett. B120, 110 (1983).

[12] K. Choi, J. E. Kim, and H. P. Nilles, Phys. Rev. Lett. 73, 1758 (1994); J. Bagger, E. Poppitz and L. Randall, Nucl. Phys. B455, 59 (1995).

[13] M. K. Gaillard, V. Jain and K. Saririan, Phys. Lett. B387, 520 (1996); K. Choi, J. S. Lee, and C. Munoz, Phys. Rev. Lett. 80, 3686 (1998).

[14] J. E. Kim, Phys. Rep. 150, 1 (1987); H. Y. Cheng, Phys. Rep. 158, 1 (1988); R. D. Peccei, in CP Violation, ed. C. Jarlskog (World Scientific, 1989).

[15] See for instance K. Choi and H. D. Kim, hep-ph/9809286.

[16] S. M. Carroll, astro-ph/9806099.

[17] J. E. Kim, hep-ph/9811509.

[18] H. Georgi and I. N. McArthur, HUTP 81/A011 (1981) (unpublished); D. B. Kaplan and A. V. Manohar, Phys. Rev. Lett. 56, 2004 (1986); K. Choi, C. W. Kim and W. K. Sze, Phys. Rev. Lett. 61, 794 (1988).

[19] P. Binetruy, hep-ph/9810553.

[20] R. Holman et al., Phys. Lett. B282, 132 (1992); M. Kamionkowski and J. March Russel, ibid. 282, 137 (1992); S. M. Barr and D. Seckel, Phys. Rev. D46, 539 (1992).

[21] H. P. Nilles, Phys. Lett. B180, 240 (1986).

[22] T. Banks and M. Dine, Nucl. Phys. B479, 173 (1996).

[23] H. P. Nilles and S. Stieberger, Nucl. Phys. B499, 3 (1997).

[24] K. Choi, Phys. Rev. D56, 6588 (1997).

[25] K. Benakli, hep-th/9805181.

[26] S. Stieberger, hep-th/9807124.

[27] K. Choi and J. E. Kim, Phys. Lett. B165, 71 (1985); L. E. Ibanez and H. P. Nilles, Phys. Lett. B169, 354 (1986); J. P. Derendinger, L. E. Ibanez and H. P. Nilles, Nucl. Phys. B267, 365 (1986). 
[28] E. Witten, Nucl. Phys. B471, 135 (1996).

[29] E. Cremmer et al., Nucl. Phys. B250, 385 (1985).

[30] J. Louis and K. Förger, hep-th/9611184; G. Curio, hep-th/9708009; D. Lüst, hepth/9803072.

[31] K. Choi, H. B. Kim and H. D. Kim, hep-th/9808122, Mod. Phys. Lett. A14, 125 (1999).

[32] M. Dine, N. Seiberg, X.-G. Wen, and E. Witten, Nucl. Phys. B289, 319 (1987); B278, 769 (1986).

[33] L. J. Dixon, V. S. Kaplunovsky and J. Louis, Nucl. Phys. B355, 649 (1991); S. Hosono, A. Klemm, S. Theisen and S.-T. Yau, Nucl. Phys. B433, 501 (1995).

[34] For a recent discussion of Type I string phenomenology with D-brane configurations, see L. E. Ibanez, C. Munoz and S. Rigolin, hep-ph/9812397.

[35] K. Choi, D. B. Kaplan and A. E. Nelson, Nucl. Phys. B391, 515 (1993); M. Dine, R. G. Leigh and D. A. MacIntire, Phys. Rev. Lett. 69, 2030 (1992).

[36] M. Dine, Y. Nir and Y. Shadmi, hep-th/9806124.

[37] L. Randall and S. Thomas, Nucl. Phys. B449, 229 (1995).

[38] K. Choi, H. D. Kim and H. C. Lee, in preparation. 


\section{FIGURES}

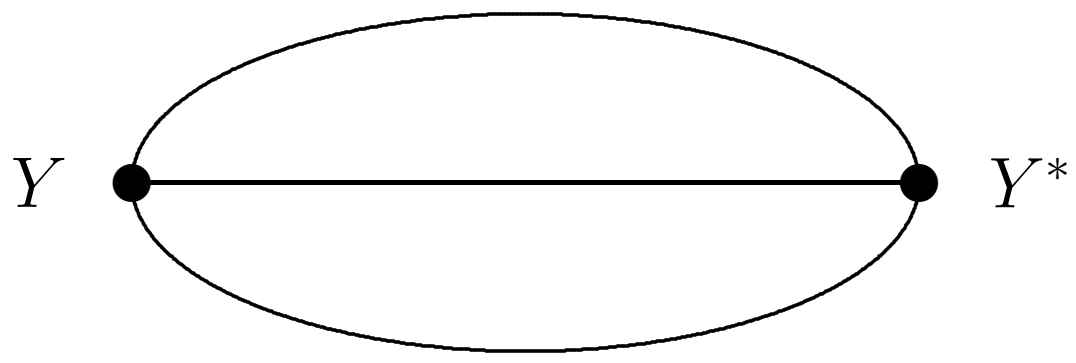

FIG. 1. Two loop supergraph inducing the quadratically-divergent potential energy of $Y_{\alpha \beta \gamma}$.

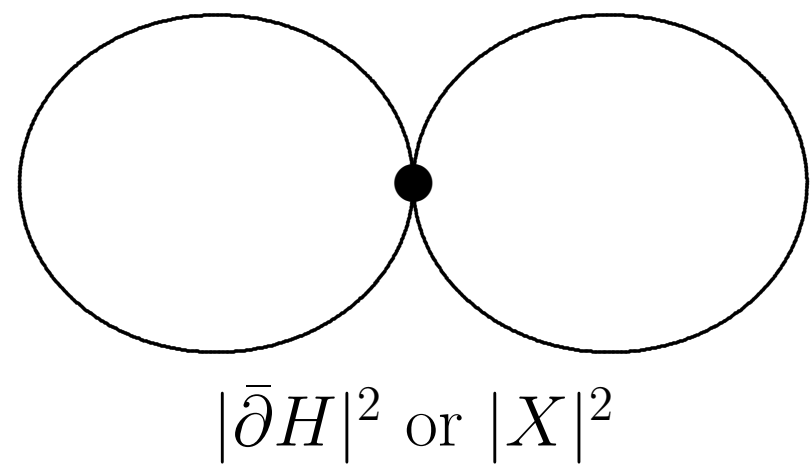

FIG. 2. Quartically-divergent two loop supergraph for the potential energy of $H_{\alpha \beta}$ and $X_{\alpha \beta \bar{\gamma}}$.

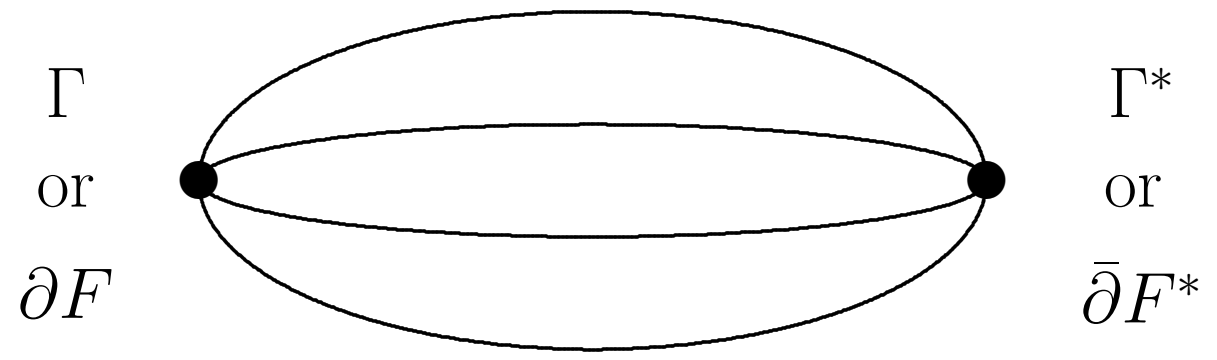

FIG. 3. Three loop supergraph for the potential energy of $\Gamma_{\alpha \beta \gamma \delta}$ and $F_{a \alpha \beta}$. For $F_{a \alpha \beta}$, two lines denote the gauge multiplets while the others are the charged matter multiplets. 